\title{
Therapeutic alternatives in the management of osteoradionecrosis of the jaws. Systematic review
}

\author{
Gisela CV Camolesi ${ }^{1}$, Karem L. Ortega ${ }^{2}$, Janaina Braga Medina ${ }^{3,4}$, Luana Campos ${ }^{5,6}$, Alejandro I Lorenzo \\ Pouso $^{7}$, Pilar Gándara Vila ${ }^{8}$, Mario Pérez Sayáns ${ }^{8}$
}

\begin{abstract}
${ }^{1}$ DDS. Assistant Professor of Specialization in Oral Maxillofacial Surgery at Foundation for Scientific and Technological Development of Dentistry, University of São Paulo, Brazil

${ }^{2}$ PhD, DDS. Department of Stomatology, School of Dentistry, University of São Paulo, Brazil

${ }^{3}$ DDS. Department of Stomatology, School of Dentistry, University of São Paulo, Brazil

${ }^{4}$ Division of Dentistry, Mario Covas State Hospital of Santo André, São Paulo, Brazil

${ }^{5} \mathrm{PhD}$, DDS. Department of Post-graduation in Implantology, University of Santo Amaro, School of Dentistry. São Paulo, Brazil

${ }^{6}$ Oral medicine, Brazilian Cancer Control Institute. São Paulo, Brazil

${ }^{7}$ DDS. Oral Medicine, Oral Surgery and Implantology Unit (MedOralRes). Faculty of Medicine and Dentistry Universidade de Santiago de Compostela, Spain

${ }^{8}$ PhD, DDS. Oral Medicine, Oral Surgery and Implantology Unit (MedOralRes). Faculty of Medicine and Dentistry Universidade de Santiago de Compostela, Spain
\end{abstract}

Correspondence:

Entrerrios $s / n$

Santiago de Compostela

C.P. 15782, Spain

perezsayans@gmail.com

Camolesi GCV, Ortega KL, Medina JB, Campos L, Lorenzo Pouso AI, Gándara Vila $\mathrm{P}$, et al. Therapeutic alternatives in the management of osteoradionecrosis of the jaws. Systematic review. Med Oral Patol Oral Cir Bucal. 2021 Mar 1;26 (2):e195-207.

\begin{tabular}{l}
\hline Article Number:24132 http://www.medicinaoral.com/ \\
C Medicina Oral S. L. C.I.F. B 96689336 - pISSN 1698-4447 - eISSN: 1698-6946 \\
eMail: medicina@medicinaoral.com \\
Indexed in: \\
Science Citation Index Expanded \\
Journal Citation Reports \\
Index Medicus, MEDLINE, PubMed \\
Scopus, Embase and Emcare \\
Indice Médico Español \\
\hline
\end{tabular}

\begin{abstract}
Background: to systematically review the literature, comparing the healing of osteoradionecrosis (ORN) among the therapeutic alternatives: surgical, pharmacological and combined.

Material and Methods: The review was organized according to the PRISMA protocol with regards to the following PICO question: patients with ORN of the jaws $(\mathrm{P}=$ Patient); all interventions reported $(\mathrm{I}=$ intervention); between all therapies $(\mathrm{C}=$ Comparison); healing of lesions $(\mathrm{O}=$ outcome $)$.

Results: Surgical treatment was the most common choice (46.3\%) followed by pharmacological treatment, exclusively (25.9\%) or combined (26.9\%). Treatment exclusively by surgical intervention seems to be most effective option, with $51.2 \%$ of the lesions healed, OR for healing of 5.7 (CI95\% 1.9-16.9, $p=0.002)$. Only 1 case ( $0.9 \%)$ corresponded to low level laser therapy.

Conclusions: It seems clear that early intervention with conservative surgical combined with pharmacological methods improves the prognosis of ORN.
\end{abstract}

Key words: Osteoradionecrosis, radiotherapy bone necrosis, hyperbaric oxygen, pentoxifylline, teriparatide, low level laser therapy. 


\section{Introduction}

Radiotherapy (RT) alone or in combination with chemotherapy or surgery is an established form of therapy for the treatment of head and neck cancer (1). Nonetheless, it has significant limitations due to its short-term (such as mucositis, dry mouth and loss of taste) and long-term (subcutaneous soft-tissue fibrosis, neck muscle atrophy, swallowing abnormality, carotid damage, trismus, radiation caries, and osteoradionecrosis (ORN) side effects $(2,3)$. Despite the use of 3D conformal RT (3D-CRT) and Intensity Modulated RT (IMRT), ORN of the jaws remains one of the most common resulting complications $(4,5)$. The reported incidence of ORN in the population of irradiated head and neck patients is rather variable, ranging from $4.7 \%$ to $37.5 \%$ and it is considered a late event, with the vast majority of cases occurring in the first 3 years following treatment $(6,7)$.

ORN can occur spontaneously due to genetic factors related to the TGF- $\beta 1$ gene (8), or it can be the result of trauma (tooth extraction and denture-related irritations are common causes). Due to its low vascular nature and thicker cortical, mandibular ORN is more common than maxillary ORN (9-13). It is defined as irradiated and exposed bone tissue which fails to heal over a period of 3 months, without the presence of a residual or recurrent tumour $(9,10,14,15)$. Although ORN can be observed without presenting bone exposure (16), normally clinically, it can range from a small area of intraoral bone exposure to extraoral fistulas and even pathological fractures. Pain, swelling, difficulties in mastication, paresthesia and facial deformities are possible sequelae of ORN and these have a significant impact on quality of life $(7,17)$. The pathogenesis of ORN remains unknown. Marx's initial proposal -the theory of hypoxia, hypovascularity and hypocellularity $(3 \mathrm{Hs})$ leading to a non-healing wound- has recently been questioned, and likewise, it has not been supported by the results of several subsequent studies $(9,18,19)$. In 2004, Delanian (20) proposed the radiation-induced fibroatrophic process (RIF) theory, which includes the formation of free radicals, endothelial dysfunction, inflammation, microvascular thrombosis, fibrosis, remodelling, and eventually bone and tissue necrosis.

The chosen treatment is based on the stage of the disease, as well as patient-related factors, however, the cure actually is not the desired outcome in the treatment of ORN, it is the abolition of symptoms and progression that is the goal. Several therapies have already been reported which have led to widespread opinions, nonetheless, there is still no universally accepted approach. More traditional or early-stage approaches include conservative treatments with oral hygiene control; hyperbaric oxygen (HBO) (prophylactically or therapeutically); the use of antibiotics over a variable period of time (although ORN is not an infectious process per se); and surgical debridement. Surgical management may be classified into minor and major procedures (21). In order to achieve satisfactory results, cases which do not respond to conservative treatment choices or those which present more advanced stages are treated with surgical resection, with or without the reconstruction of vascularised tissue(21). All of these treatments were guided mainly by Marx's theory (9). More recently and in light of the pathophysiology of the disease proposed by Delanian (20), pharmacological treatment with pentoxifylline-tocopherol with or without clodronate (PENTOCLO) (22), teriparatide (23) and low-level laser therapy (LLLT) (24) have been introduced.

Therefore, the aim of this paper is to systematically review the literature, comparing the healing of ORN with all the reported therapies: surgical, pharmacological and combined.

\section{Material and Methods}

- Protocol and registration

The design of this study was registered in PROSPERO (Ref. 159983). This review was carried out following the PRISMA guidelines and according to the PICO method (25): patients with ORN of the jaws ( $\mathrm{P}=$ Patient); all interventions related $(\mathrm{I}=$ intervention); between all therapies $(\mathrm{C}=$ Comparison $)$; healing of lesions $(\mathrm{O}=$ outcome $)$. - Selection criteria, sources of information and search We conducted a bibliographic search in PubMed, Web of Science, Scopus, LiLACS, OVID, EMBASE, Cochrane Library, Clinical Trials, the five WHO regional bibliographic databases (AIM, LILACS, IMEMR, IMSEAR, WPRIM), and the Conference Proceedings Citation Index in order to identify relevant studies on ORN of the jaws between the first records found in the database and November 2019.

Inclusion criteria: All of the articles on case series, case reports, cohort studies, and case and control studies with no language limitation were included.

Exclusion criteria: Articles which do not deal with RTinduced osteonecrosis; unavailable abstract; complete maxillectomy; other systematic reviews; studies that have not been conducted on humans.

Selection of studies: Two independent researchers, MPS and GCVC, analysed the abstracts of the articles obtained in the search which had met the search criteria, that is to say, texts that dealt with patients with ORN of the jaws and their management. Both of the researchers subsequently read the full article in order to determine whether or not it met the inclusion criteria. A third researcher, LC, acted as a mediator in the case of any disputes. Data collection process: Data from all the articles was collected by both researchers independently (in duplicate) and this data was corroborated by the third party who acted as a mediator in case of discrepancy or lack of agreement. 
- Study variables

The following information was extracted from each study: First author, year of publication, type of study, location of cancerous lesion, dose used in RT, management of the lesion (surgical, pharmacological or combined), location (maxilla, jaw), region (anterior, posterior), quantity (single, multiple), and also the time from the end of RT to the diagnosis of ORN, time until healing, maximum follow-up time, and finally whether or not there were any recurrences.

- Risk of bias

The methodological quality and the risk of bias of the included studies were assessed using the Newcastle-Ottawa scale (NOS)(26). For studies, cohorts and cases and controls, which amounted to $4.6 \%$ of the included studies, the original NOS scale was used, and for the remaining $95.4 \%$, that is to say, the case series and case report studies, Pierson and Bradford Hills' modified NOS scale (27) was used. This analysis was carried out independently by each of the two researchers and in the case of any disagreements the third researcher acted as a mediator. - Statistical analysis

All of the variables were collected in a database and were analysed with SPSS v. 24.0 (IBM Inc., Madrid, Spain). Basic descriptive statistics were used for the uni- variate description, these included the mean, standard deviation, frequency and percentage. The relationship between the different categorical variables and healing was evaluated using Pearson's Chi-square. The relationship between the healing and the type of treatment and the quantitative variables was studied by using the ANOVA test to compare the means. The influence of the treatment type on the progression of ORN was assessed by using a univariate logistic regression analysis. The significance level was established at $p \leq 0.05$.

\section{Results}

The search process involved a total of 3,861 articles. After removing duplicates, 2,722 articles remained; of these 1,769 were subsequently excluded because they did not meet the inclusion criteria (Fig. 1). After fully reading the 542 articles, it was determined that 110 studies met all of the inclusion criteria and these were included.

Four of the articles were rated as high quality (3.6\%), 103 as medium quality (93.6\%), and 3 as low quality $(2.7 \%)$ (Table 1). The summary of the data of all of the patients that was extracted from the studies is depicted in Table 2, and the full descriptive results can be found in Table 3.
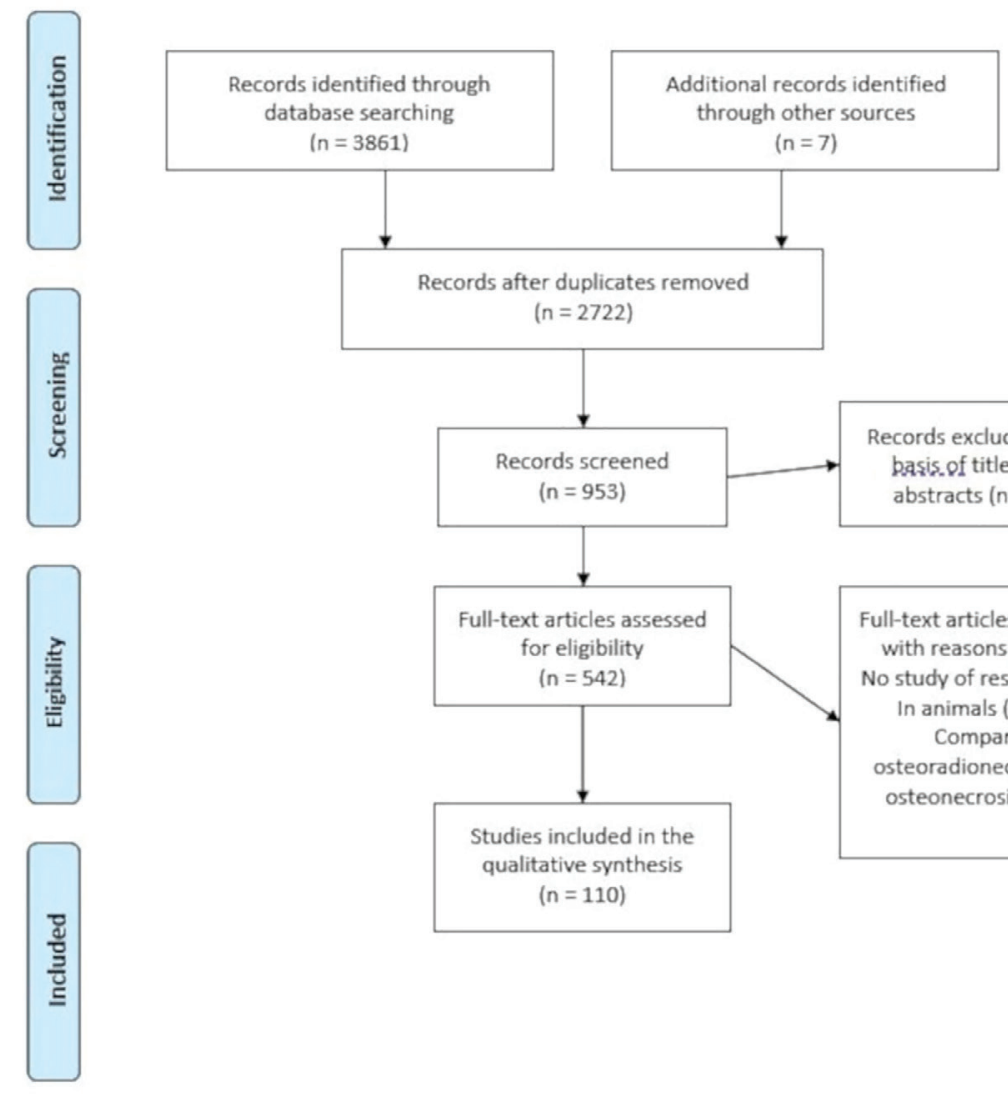

Records after duplicates removed ( $n=2722$ )

Fig. 1: PRISMA flow diagram. 
Table 1: Classification of the studies in terms of risk of bias according to the NOS scale.

\begin{tabular}{|c|c|c|c|}
\hline Author & Year & Number of Patients & Assessment \\
\hline Alam et al. & 2009 & 33 & Medium \\
\hline Ang et al. & 2003 & 21 & Medium \\
\hline Baron et al. & 2016 & 5 & Medium \\
\hline Baumann et al. & 2010 & 63 & Medium \\
\hline Beech et al. & 2016 & 1 & Medium \\
\hline Bettoni et al. & 2019 & 11 & Medium \\
\hline Bettoni et al. & 2019 & 49 & Medium \\
\hline Bianco et al. & 2019 & 8 & Medium \\
\hline Bohn et al. & 2015 & 3 & Medium \\
\hline Bouguila et al. & 2015 & 22 & Medium \\
\hline Breik et al. & 2019 & 2 & Medium \\
\hline Cannady et al. & 2010 & 53 & Medium \\
\hline Cha et al. & 2018 & 2 & Medium \\
\hline Chandarana et al. & 2013 & 12 & Medium \\
\hline Chang et al. & 2001 & 29 & Medium \\
\hline Chang et al. & 2011 & 35 & High \\
\hline Chen et al. & 2014 & 153 & Medium \\
\hline Chen et al. & 2016 & 105 & High \\
\hline Chen et al. & 2018 & 1 & Medium \\
\hline Chen et al. & 2019 & 1 & Medium \\
\hline Chiapasco et al. & 2006 & 59 & Medium \\
\hline Choi et al. & 2014 & 1 & medium \\
\hline Chronopoulos et al. & 2015 & 115 & Medium \\
\hline Coskunfirat et al. & 2004 & 12 & Medium \\
\hline Curi et al. & 1997 & 104 & Medium \\
\hline Curi et al. & 2000 & 18 & Medium \\
\hline Curi et al. & 2007 & 5 & Medium \\
\hline D'Hauthuille et al. & 2008 & 59 & Medium \\
\hline Dai et al. & 2015 & 120 & Medium \\
\hline Danielsson et al. & 2019 & 17 & Medium \\
\hline David et al. & 2001 & 51 & Medium \\
\hline De Felice et al. & 2016 & 36 & Medium \\
\hline Delanian et al. & 2005 & 18 & Medium \\
\hline Delanian et al. & 2011 & 54 & Medium \\
\hline Dieleman et al. & 2017 & 27 & Medium \\
\hline Dissard et al. & 2019 & 27 & Medium \\
\hline D'Souza et al. & 2007 & 23 & Medium \\
\hline D'Souza et al. & 2009 & 58 & Low \\
\hline D'Souza et al. & 2014 & 71 & Medium \\
\hline \begin{tabular}{|l} 
Epstein et al. \\
\end{tabular} & 1997 & 26 & Medium \\
\hline Etezadi et al. & 2013 & 1 & Medium \\
\hline \begin{tabular}{|l} 
Fan et al. \\
\end{tabular} & 2016 & 31 & Medium \\
\hline Freiberger et al. & 2009 & 65 & Medium \\
\hline Gal et al. & 2003 & 30 & Medium \\
\hline Gallegos et al. & 2015 & 25 & Medium \\
\hline Gallesio et al. & 2015 & 10 & Medium \\
\hline Gavriel et al. & 2017 & 21 & Medium \\
\hline Gevorgyan et al. & 2013 & 14 & Medium \\
\hline Gupta et al. & 2013 & 33 & Medium \\
\hline Haffey et al. & 2019 & 8 & Medium \\
\hline Hamilton et al. & 2012 & 14 & Medium \\
\hline Harris M et al. & 1992 & 24 & Medium \\
\hline Hayashi et al. & 2015 & 13 & Medium \\
\hline Hirsch et al. & 2008 & 305 & High \\
\hline
\end{tabular}


Table 1 cont.: Classification of the studies in terms of risk of bias according to the NOS scale.

\begin{tabular}{|c|c|c|c|}
\hline Ioannides et al. & 1994 & 28 & Medium \\
\hline Jacobson et al. & 2010 & 1 & Medium \\
\hline Jenwitheesuk et al. & 2018 & 84 & Medium \\
\hline Jisander et al. & 1999 & 8 & Medium \\
\hline Kahenasa et al. & 2012 & 1 & Medium \\
\hline Kildal et al. & 2001 & 1 & Medium \\
\hline Kim et al. & 2016 & 8 & Medium \\
\hline Kobayashi et al. & 2000 & 4 & Medium \\
\hline Kraeima et al. & 2018 & 3 & Medium \\
\hline Kumar et al. & 2018 & 25 & Low \\
\hline LaDow C.S et al. & 1950 & 1 & Medium \\
\hline Lyons et al. & 2013 & 30 & Medium \\
\hline Magremanne M et al. & 2018 & 1 & Medium \\
\hline Mainous et al. & 2015 & 1 & Low \\
\hline Mainous et al. & 2014 & 2 & Medium \\
\hline Man et al. & 1975 & 14 & Medium \\
\hline Manimaran et al. & 1973 & 1 & Medium \\
\hline Manzano et al. & 2019 & 20 & Medium \\
\hline Manzon et al. & 2015 & 2 & Medium \\
\hline Mao et al. & 2004 & 11 & Medium \\
\hline Marwan et al. & 1983 & 58 & Medium \\
\hline Marx RE et al. & 2017 & 150 & Medium \\
\hline Mc Leod et al. & 2012 & 12 & Low \\
\hline Milani et al. & 2019 & 1 & Medium \\
\hline Militsakh et al. & 2005 & 9 & Medium \\
\hline Moran et al. & 1987 & 1 & Medium \\
\hline Mounsey et al. & 1993 & 41 & Medium \\
\hline Mücke et al. & 2013 & 94 & Medium \\
\hline Nabil et al. & 2012 & 10 & Medium \\
\hline Nakatsuka et al. & 1996 & 9 & Medium \\
\hline Notani et al. & 2003 & 87 & Medium \\
\hline Oh et al. & 2009 & 114 & Medium \\
\hline Ohba et al. & 2013 & 12 & Medium \\
\hline Patel et al. & 2016 & 62 & Medium \\
\hline Piccin et al. & 2016 & 1 & Medium \\
\hline Pinto et al. & 2017 & 21 & High \\
\hline Porcaro et al. & 2015 & 1 & Medium \\
\hline Reuther et al. & 2003 & 68 & Medium \\
\hline Ribeiro et al. & 2018 & 20 & Medium \\
\hline Robard et al. & 2014 & 27 & Medium \\
\hline Rommel et al. & 2018 & 15 & Medium \\
\hline Santamaria et al. & 1998 & 12 & Medium \\
\hline Scala et al. & 2010 & 1 & Medium \\
\hline Shaha et al. & 1998 & 6 & Medium \\
\hline Shan et al. & 2015 & 5 & Medium \\
\hline Shimizu et al. & 2012 & 2 & Medium \\
\hline Southerland et al. & 1993 & 1 & Medium \\
\hline Suh et al. & 2010 & 40 & Medium \\
\hline Sullivan et al. & 1989 & 17 & Medium \\
\hline Teixeira et al. & 1991 & 8 & Medium \\
\hline Van Merkesteyn et al & 1994 & 1 & Medium \\
\hline Van Merkesteyn et al. & 1995 & 29 & Medium \\
\hline Vudiniabola et al. & 2000 & 14 & Medium \\
\hline Wong et al. & 1997 & 32 & Medium \\
\hline Woo et al. & 2016 & 1 & Medium \\
\hline Young et al. & 2016 & 4 & Medium \\
\hline
\end{tabular}


Table 2: Descriptive summary of all of the articles.

\begin{tabular}{|c|c|c|c|c|c|c|c|c|c|c|c|}
\hline Author & Year & $\begin{array}{l}\text { Location of } \\
\text { the cancer- } \\
\text { ous lesion }\end{array}$ & $\begin{array}{l}\text { RT } \\
\text { dose } \\
\text { (Gy) }\end{array}$ & $\begin{array}{l}\text { ORN manage- } \\
\text { ment }\end{array}$ & $\begin{array}{l}\text { ORN } \\
\text { loca- } \\
\text { tion }\end{array}$ & ORN region & $\begin{array}{c}\text { ORN } \\
\text { lesions }\end{array}$ & $\begin{array}{c}\text { Time } \\
\text { from } \\
\text { RT to } \\
\text { ORN } \\
\text { months }\end{array}$ & $\begin{array}{c}\text { Heal- } \\
\text { ing }\end{array}$ & $\begin{array}{l}\text { Follow- } \\
\text { up time } \\
\text { months }\end{array}$ & $\begin{array}{l}\text { ORN } \\
\text { recur- } \\
\text { rences }\end{array}$ \\
\hline Alam et al. & 2009 & $1,3,5,6,9$ & - & Surgical & Jaw & Anterior and Posterior & Multiple & 47 & No & - & Yes \\
\hline Ang et al. & 2003 & $3,6,7,9,10$ & 60 & Combined & Multiple & - & Single & 48 & No & 79 & Yes \\
\hline Baron et al. & 2016 & $12,1,9,4,2$ & - & Surgical & Jaw & Anterior and Posterior & Multiple & 63.6 & Yes & 48 & No \\
\hline Baumann et al. & 2011 & - & 66.5 & Surgical & Jaw & Anterior and Posterior & Multiple & - & Yes & 72 & No \\
\hline Beech et al. & 2016 & 2 & - & Surgical & Jaw & Posterior & Single & 60 & Yes & - & No \\
\hline Bettoni et al. & 2019 & $1,13,8,7,2$ & - & Surgical & Jaw & - & Single & 48.5 & Yes & 83 & No \\
\hline Bettoni et al. & 2019 & - & 63.6 & Combined & Jaw & Anterior and Posterior & Multiple & 57 & No & - & Yes \\
\hline Bianco et al. & 2019 & $7,14,9,15,16,8$ & 78 & Pharmacological & Multiple & - & Multiple & 23.2 & Yes & 12 & No \\
\hline Bohn et al. & 2016 & $4,13,2$ & 55.4 & Pharmacological & Jaw & Anterior and Posterior & Multiple & 72 & Yes & 12 & No \\
\hline Bouguila et al. & 2015 & 5,13 & 72 & Combined & - & - & - & 48 & Yes & - & No \\
\hline Breik et al. & 2019 & 9,2 & 70 & Pharmacological & Jaw & Posterior & Single & 10 & Yes & - & No \\
\hline Cannady et al. & 2011 & 3,8 & - & Surgical & Multiple & - & Single & - & Yes & - & No \\
\hline Cha et al. & 2018 & 2 & 60 & Pharmacological & Jaw & Posterior & Single & 180 & Yes & - & No \\
\hline $\begin{array}{c}\text { Chandarana } \\
\text { et al. }\end{array}$ & 2013 & - & - & Surgical & - & - & - & - & Yes & - & No \\
\hline Chang et al. & 2001 & $\begin{array}{c}8,2,9,7,4 \\
16,18,15\end{array}$ & 67.7 & Surgical & Jaw & - & Single & - & Yes & - & No \\
\hline Chang et al. & 2011 & $\begin{array}{l}13,20,4,2 \\
19,16,15,9\end{array}$ & 67.4 & Surgical & Jaw & - & Single & 46 & Yes & 36 & No \\
\hline Chen et al. & 2014 & - & 87.4 & Surgical & Jaw & - & Single & 29.8 & Yes & - & No \\
\hline Chen et al. & 2016 & $\begin{array}{l}13,20,4,2, \\
19,16,15,9\end{array}$ & 74 & Combined & Jaw & Posterior & Multiple & 72 & Yes & 8 & No \\
\hline Chen et al. & 2018 & 5 & - & Surgical & - & - & - & - & Yes & - & No \\
\hline Chen et al. & 2019 & 2 & 72 & Pharmacological & Multiple & Anterior and Posterior & Multiple & 60 & Yes & - & No \\
\hline $\begin{array}{c}\text { Chiapasco } \\
\text { et al. }\end{array}$ & 2006 & $11,2,4,10$ & 53.5 & Surgical & Multiple & Anterior and Posterior & Single & - & Yes & 120 & No \\
\hline Choi et al. & 2014 & 9 & - & Surgical & Multiple & - & Single & 84 & Yes & - & No \\
\hline $\begin{array}{c}\text { Chronopoulos } \\
\text { et al. }\end{array}$ & 2015 & $4,2,6$ & 63.4 & Combined & Jaw & Anterior and Posterior & Single & - & No & - & Yes \\
\hline $\begin{array}{l}\text { Coskunfirat } \\
\text { et al. }\end{array}$ & 2004 & $5,3,10,2,21$ & 65 & Surgical & Multiple & - & Multiple & - & Yes & 62 & No \\
\hline Curi et al. & 1997 & $\begin{array}{c}13,2,20,4 \\
16,15,8,5,6 \\
7,22\end{array}$ & 60 & Combined & Multiple & - & Single & 18 & No & 12 & Yes \\
\hline Curi et al. & 2000 & $\begin{array}{l}13,2,20,4 \\
16,15,8,9\end{array}$ & 6.2 & Pharmacological & Multiple & - & Single & 27.9 & Yes & 24.8 & No \\
\hline Curi et al. & 2007 & $2,16,4$ & 65 & Surgical & Jaw & Anterior and Posterior & Single & 45.6 & Yes & - & No \\
\hline $\begin{array}{l}\text { D'Hauthuille } \\
\text { et al. }\end{array}$ & 2008 & - & - & Surgical & Jaw & Anterior and Posterior & Single & - & Yes & - & No \\
\hline Dai et al. & 2015 & $\begin{array}{c}2,23,4,11, \\
10,20,16, \\
6,22\end{array}$ & 68.1 & Surgical & Multiple & Anterior and Posterior & Multiple & 36 & No & - & Yes \\
\hline $\begin{array}{l}\text { Danielsson } \\
\text { et al. }\end{array}$ & 2019 & $9,1,2,19,12$ & 68 & Surgical & Jaw & - & Single & 37.2 & No & 12 & Yes \\
\hline David et al. & 2001 & $\begin{array}{c}2,4,16,8,19 \\
5,11\end{array}$ & 51.8 & Pharmacological & Jaw & - & Single & 32 & No & 108 & Yes \\
\hline $\begin{array}{c}\text { De Felice et } \\
\text { al. }\end{array}$ & 2016 & $\begin{array}{c}3,8,7,22 \\
28,29\end{array}$ & 66.3 & Combined & Multiple & Anterior and Posterior & Multiple & 6 & No & - & Yes \\
\hline Delanian et al. & 2005 & 3,8 & 65 & Pharmacological & Jaw & Posterior & Single & 7.25 & Yes & - & No \\
\hline Delanian et al. & 2011 & 3,8 & 62.5 & Pharmacological & Jaw & - & Single & 15.5 & Yes & 36 & No \\
\hline Dieleman et al. & 2017 & $4,1,2,15,14$ & 60 & Combined & Jaw & - & - & 36 & Yes & 24 & No \\
\hline Dissard et al. & 2019 & $2,4,3,8,18$ & 65 & Pharmacological & Jaw & Anterior and Posterior & Multiple & 87.5 & Yes & 24 & No \\
\hline
\end{tabular}


Table 2 cont.: Descriptive summary of all of the articles.

\begin{tabular}{|c|c|c|c|c|c|c|c|c|c|c|c|}
\hline D'Souza et al. & 2007 & $3,8,7,18$ & 64 & Combined & Jaw & - & Single & 48 & Yes & 30 & No \\
\hline D'Souza et al. & 2009 & - & - & Pharmacological & - & - & - & - & Yes & - & No \\
\hline D'Souza et al. & 2014 & $3,8,7,18$ & 64 & Combined & Jaw & - & Single & 25 & Yes & 69 & No \\
\hline Epstein et al. & 1997 & 9 & - & Combined & - & - & - & 50 & No & 123 & Yes \\
\hline Etezadi et al. & 2013 & - & 70 & Surgical & Jaw & Posterior & Multiple & - & Yes & - & No \\
\hline Fan et al. & 2016 & $\begin{array}{c}5,4,15,2 \\
17,8,1,9,16 \\
7,22\end{array}$ & 76.3 & Surgical & Jaw & - & - & - & Yes & 72 & No \\
\hline $\begin{array}{l}\text { Freiberger } \\
\text { et al. }\end{array}$ & 2009 & $1,12,18$ & 67.5 & Combined & Jaw & Anterior and Posterior & Multiple & 73.3 & Yes & 56 & No \\
\hline Gal et al. & 2003 & - & - & Combined & Jaw & - & Single & - & No & - & Yes \\
\hline Gallegos et al. & 2015 & $2,9,7,8$ & - & Pharmacological & Jaw & Posterior & Single & 24 & No & 36 & Yes \\
\hline Gallesio et al. & 2015 & $\begin{array}{c}22,14,16, \\
28,30\end{array}$ & - & Surgical & Multiple & - & Single & - & Yes & 12 & No \\
\hline Gavriel et al. & 2017 & $\begin{array}{c}9,2,15,18 \\
16,19\end{array}$ & 53 & Pharmacological & Multiple & - & Single & 12 & Yes & 25.2 & No \\
\hline $\begin{array}{l}\text { Gevorgyan } \\
\text { et al. }\end{array}$ & 2013 & $\begin{array}{c}2,4,16,7,8, \\
\text { alveolus }\end{array}$ & - & Combined & Jaw & - & Single & 26.9 & No & 26 & Yes \\
\hline Gupta et al. & 2013 & $\mathrm{~N} / \mathrm{R}$ & 60 & Pharmacological & Jaw & - & Single & 7.5 & Yes & 12 & No \\
\hline Haffey et al. & 2019 & $2,1,9,18$ & - & Surgical & Jaw & Posterior & Multiple & - & Yes & 63 & No \\
\hline $\begin{array}{l}\text { Hamilton et } \\
\text { al. }\end{array}$ & 2012 & - & 65 & Combined & Jaw & Posterior & Multiple & 19.8 & Yes & - & No \\
\hline Harris M et al. & 1992 & - & 61.9 & Surgical & Jaw & Anterior and Posterior & Single & - & Yes & - & No \\
\hline Hayashi et al. & 2015 & $\begin{array}{c}2,4,16,3, \\
11,20,8,5, \\
18,12\end{array}$ & 60 & Pharmacological & Multiple & Anterior and Posterior & Multiple & - & No & - & Yes \\
\hline Hirsch et al. & 2008 & - & 66.5 & Surgical & Jaw & Anterior and Posterior & Single & - & Yes & - & No \\
\hline $\begin{array}{l}\text { Ioannides } \\
\text { et al. }\end{array}$ & 1994 & $2,4,9,20,18$ & 65 & Surgical & Jaw & Anterior and Posterior & Multiple & - & Yes & 84 & No \\
\hline Jacobson et al. & 2010 & - & - & Surgical & Jaw & Posterior & Multiple & - & Yes & - & No \\
\hline $\begin{array}{c}\text { Jenwitheesuk } \\
\text { et al. }\end{array}$ & 2018 & $5,3,23$ & - & Pharmacological & Jaw & - & Multiple & - & Yes & 6 & No \\
\hline Jisander et al. & 1999 & - & 62.5 & Combined & Jaw & Posterior & Multiple & 119 & Yes & 93 & No \\
\hline $\begin{array}{c}\text { Kahenasa } \\
\text { et al. }\end{array}$ & 2012 & 9,1 & 70 & Pharmacological & Jaw & Posterior & Single & 6 & Yes & - & No \\
\hline Kildal et al. & 2001 & 28 & 72 & Surgical & Jaw & Posterior & Multiple & 84 & Yes & - & No \\
\hline Kimet al. & 2016 & $5,19,12,18$ & 71 & Surgical & Jaw & Anterior and Posterior & Single & - & Yes & 85 & No \\
\hline $\begin{array}{l}\text { Kobayashi } \\
\text { et al. }\end{array}$ & 2000 & $2,19,8$ & 90 & Surgical & Jaw & Posterior & Single & - & Yes & 41 & No \\
\hline Kraeima et al. & 2018 & 4 & 61 & Combined & Jaw & Posterior & Single & 11.5 & No & - & Yes \\
\hline Kumar et al. & 2018 & $\begin{array}{c}1,4,9,2,11 \\
7,10\end{array}$ & 62 & Surgical & Multiple & Anterior and Posterior & Single & 48 & Yes & - & No \\
\hline $\begin{array}{c}\text { LaDow C.S } \\
\text { et al. }\end{array}$ & 1950 & 9 & 51 & Surgical & Jaw & Posterior & Single & 36 & Yes & - & No \\
\hline Lyons et al. & 2013 & $\begin{array}{c}4,2,16,31, \\
14,10,3,8, \\
18,15\end{array}$ & 62 & Surgical & Multiple & - & - & - & No & - & No \\
\hline $\begin{array}{l}\text { Magremanne } \\
\text { M et al. }\end{array}$ & 2018 & 8 & 70 & Pharmacological & Jaw & Posterior & Single & 42 & Yes & 6 & No \\
\hline Mainous et al. & 1973 & 4 & 80 & Pharmacological & Jaw & Anterior and Posterior & Single & 17 & Yes & - & No \\
\hline Mainous et al. & 1974 & $2,4,20,9,15$ & 70 & Pharmacological & Jaw & Anterior and Posterior & Single & - & Yes & - & No \\
\hline Man et al. & 2015 & 14,11 & 60 & Surgical & Jaw & - & Single & - & Yes & - & No \\
\hline $\begin{array}{c}\text { Manimaran } \\
\text { et al. }\end{array}$ & 2014 & 16,9 & - & Combined & Jaw & Posterior & Single & 36 & Yes & 24 & No \\
\hline Manzano et al. & 2019 & $\begin{array}{c}4,5,14,19 \\
8,1\end{array}$ & 60.1 & Combined & Multiple & Anterior and Posterior & Multiple & 6.5 & No & - & Yes \\
\hline Manzon et al. & 2015 & 11 & 55 & Surgical & Jaw & - & Single & - & Yes & - & No \\
\hline
\end{tabular}


Table 2 cont.: Descriptive summary of all of the articles.

\begin{tabular}{|c|c|c|c|c|c|c|c|c|c|c|c|}
\hline Mao et al. & 2004 & - & - & Surgical & - & - & - & - & Yes & - & No \\
\hline Marwan et al. & 2017 & $1,8,4,23$ & - & Surgical & - & - & - & - & Yes & - & No \\
\hline $\begin{array}{l}\text { Marx RE } \\
\text { et al. }\end{array}$ & 1983 & - & - & Combined & Jaw & Anterior and Posterior & Single & - & Yes & - & No \\
\hline Mc Leod et al. & 2012 & 2 & - & Pharmacological & - & - & Single & 40 & Yes & - & No \\
\hline Milani et al. & 2019 & $23,3,8,29$ & - & Pharmacological & Jaw & Posterior & Single & 60 & Yes & 12 & No \\
\hline $\begin{array}{l}\text { Militsakh } \\
\text { et al. }\end{array}$ & 2005 & 1 & 71 & Surgical & Jaw & Anterior and Posterior & Single & - & Yes & 67 & No \\
\hline Moran et al. & 1987 & $2,4,19$ & 55 & Surgical & Jaw & Anterior and Posterior & Single & 14 & Yes & - & No \\
\hline Mounsey et al. & 1993 & $4,2,10$ & 47.5 & Pharmacological & Jaw & Anterior and Posterior & Multiple & 39 & No & - & Yes \\
\hline Mücke et al. & 2013 & 5,2 & 69.3 & Combined & Multiple & - & Multiple & - & No & 12 & Yes \\
\hline Nabil et al. & 2012 & 2,8 & - & Surgical & Multiple & Anterior and Posterior & Multiple & - & No & 48 & Yes \\
\hline $\begin{array}{c}\text { Nakatsuka } \\
\text { et al. }\end{array}$ & 1996 & $\begin{array}{c}2,22,20 \\
13,19 \\
\end{array}$ & 80 & Surgical & Jaw & Anterior and Posterior & Multiple & - & Yes & 61 & No \\
\hline Notani et al. & 2003 & $\begin{array}{c}2,4,15,9,19 \\
14,6,5,121 \\
18,13\end{array}$ & 90 & Combined & Jaw & - & Single & - & Yes & 444 & No \\
\hline Oh et al. & 2009 & $2,8,16,10$ & 8.4 & Combined & Jaw & - & Single & 33 & No & 372 & Yes \\
\hline Ohba et al. & 2013 & $8,3,6,5$ & 64 & Pharmacological & Jaw & Anterior and Posterior & Single & 13 & Yes & 30.3 & No \\
\hline Patel et al. & 2016 & 8 & - & Pharmacological & Jaw & - & Single & - & Yes & - & No \\
\hline Piccin et al. & 2016 & $4,2,8,15$ & 70 & Combined & Jaw & Posterior & Single & - & Yes & - & No \\
\hline Pinto et al. & 2017 & 16 & 65.6 & Surgical & Jaw & - & Single & - & No & 40 & Yes \\
\hline Porcaro et al. & 2015 & $\begin{array}{c}2,4,14,9,16 \\
7,13,28\end{array}$ & 90 & Surgical & Maxilla & Posterior & Single & 24 & No & 12 & Yes \\
\hline Reuther et al. & 2003 & - & 60 & Combined & Multiple & - & Single & 13 & No & - & Yes \\
\hline Ribeiro et al. & 2018 & 3,8 & 72 & - & Multiple & Anterior and Posterior & Single & 24 & Yes & - & No \\
\hline Robard et al. & 2014 & $\begin{array}{c}4,27,11,9 \\
23,8,2\end{array}$ & 95 & Surgical & Jaw & Anterior and Posterior & Single & 60 & Yes & - & No \\
\hline Rommel et al. & 2018 & $\begin{array}{c}15,16,5,12 \\
2,4,19 \\
\end{array}$ & - & Surgical & Jaw & - & - & - & Yes & - & No \\
\hline $\begin{array}{l}\text { Santamaria } \\
\text { et al. }\end{array}$ & 1998 & 2 & $\begin{array}{l}60 . \\
35 \\
\end{array}$ & Surgical & Jaw & Anterior and Posterior & Single & 13 & Yes & 45 & No \\
\hline Scala et al. & 2010 & $2,1,4$ & 66 & Pharmacological & Jaw & - & Multiple & 48 & Yes & 24 & No \\
\hline Shaha et al. & 1998 & $5,8,2$ & 69.5 & Surgical & Jaw & Anterior and Posterior & Single & 104.5 & Yes & 80 & No \\
\hline Shan et al. & 2015 & 5 & 66 & Surgical & Jaw & Posterior & Multiple & 104.5 & Yes & 90 & No \\
\hline Shimizu et al. & 2012 & 2,4 & 63 & Surgical & Jaw & Anterior and Posterior & Single & 48 & Yes & - & No \\
\hline $\begin{array}{l}\text { Southerland } \\
\text { et al. }\end{array}$ & 1993 & - & 84 & Combined & Jaw & Posterior & Multiple & - & Yes & - & No \\
\hline Suh et al. & 2010 & - & - & Surgical & Jaw & - & Single & - & No & 17.4 & Yes \\
\hline Sullivan et al. & 1989 & $2,1,17$ & - & Surgical & Jaw & - & - & - & Yes & 15 & No \\
\hline Teixeira et al. & 1991 & 18 & 78.3 & Combined & Jaw & - & Single & - & Yes & - & Yes \\
\hline $\begin{array}{c}\text { Van Mer- } \\
\text { kesteyn et al }\end{array}$ & 1994 & $\begin{array}{c}4,2,16,15 \\
19,9,5,13, \\
18,26 \\
\end{array}$ & 67 & Combined & Jaw & Posterior & Multiple & 2 & Yes & 6.5 & No \\
\hline $\begin{array}{c}\text { Van Mer- } \\
\text { kesteyn } \text { et al. }\end{array}$ & 1995 & - & 75 & Combined & Jaw & - & Single & 72 & No & 84 & Yes \\
\hline $\begin{array}{l}\text { Vudiniabola } \\
\text { et al. }\end{array}$ & 2000 & $\begin{array}{c}4,2,20,14, \\
16,9,15,8, \\
24,7,25 \\
\end{array}$ & 58 & Combined & Multiple & - & Single & 183 & Yes & 156 & No \\
\hline Wong et al. & 1997 & 9 & 64 & Pharmacological & Multiple & Anterior and Posterior & Single & - & Yes & 36 & No \\
\hline Woo et al. & 2016 & - & 72 & Surgical & Jaw & Posterior & Single & - & Yes & - & No \\
\hline Young et al. & 2016 & - & - & Pharmacological & - & - & - & - & Yes & - & No \\
\hline
\end{tabular}

Location of the cancerous lesion: 1.Base of the Tongue, 2.Tongue, 3.Oral cavity, 4. Floor of the mouth, 5.Nasopharynx, 6.Hypopharynx, 7.Larynx, 8.Oropharynx, 9.Amygdalin fossa, 10.Maxilla, 11.Jaw, 12.Submandibular gland, 13.Lips, 14.Alveolar ridge, 15.Retromolar trigone, 16.Soft palate, 17.Hard palate, 18.Parotid gland, 19.Oral mucosa, 20.Gingiva, 21.Nasolacrimal conduct, 22.Maxillar sinus, 23.Cheek, 24.Epiglottis, 25.Pyriform sinus, 26.Sublingual gland, 27.Uvula, 28.Minor salivary glands, 29.Major salivary glands, 30.Thyroid, 31. Tonsil. 
Table 3: Descriptive summary of extracted categorical variables.

\begin{tabular}{|c|c|c|c|}
\hline \multicolumn{2}{|l|}{ Variable } & \multirow{2}{*}{$\begin{array}{c}\mathbf{N} \\
109\end{array}$} & \multirow{2}{*}{$\begin{array}{c}\mathbf{\%} \\
99.08\end{array}$} \\
\hline & Surgical medical treatment & & \\
\hline 116 atintil givup & LLLT & 001 & 0.92 \\
\hline \multirow{3}{*}{ Treatment type } & SURGICAL & 051 & 46.8 \\
\hline & PHARMACOLOGICAL & 028 & 25.6 \\
\hline & COMBINED & 030 & 27.6 \\
\hline \multirow{7}{*}{ Surgical treatment } & FREE FLAP & 053 & 60.2 \\
\hline & PEDICULATED SOFT TISSUE & 006 & 6.8 \\
\hline & ARTIFICIAL DERMAL SKIN & 001 & 1.1 \\
\hline & DEBRIDEMENT & 020 & 22.7 \\
\hline & BLOCK GRAFT & 001 & 1.1 \\
\hline & SEGMENTAL OSTEOTOMY & 007 & 7.9 \\
\hline & Total & 088 & 100.0 \\
\hline \multirow{9}{*}{$\begin{array}{l}\text { Pharmacological treatment } \\
\text { and hyperbaric medicine }\end{array}$} & PENTOCLO & 005 & 8.5 \\
\hline & PENTOXIFYLLINE & 006 & 10.2 \\
\hline & ANTIBIOTICS AND ANTISEPTICS ALONE & 002 & 3.4 \\
\hline & PRGF & 005 & 8.5 \\
\hline & HBO & 033 & 55.9 \\
\hline & OZOSAN & 002 & 3.4 \\
\hline & TERIPARATIDE & 002 & 3.4 \\
\hline & COMBINED (2 OR MORE) & 003 & 5.2 \\
\hline & Total & 058 & 100.0 \\
\hline \multirow{5}{*}{ ORN Location } & JAW & 079 & 71.8 \\
\hline & MAXILLA & 002 & 1.8 \\
\hline & MULTIPLE & 020 & 18.2 \\
\hline & NOT SPECIFIED & 009 & 8.2 \\
\hline & Total & 110 & 100.0 \\
\hline \multirow{4}{*}{ Mandibular region } & POSTERIOR & 025 & 22.7 \\
\hline & ANTERIOR-POSTERIOR & 037 & 33.6 \\
\hline & NOT SPECIFIED & 048 & 43.6 \\
\hline & Total & 110 & 100.0 \\
\hline \multirow{4}{*}{ Number of lesions } & SINGLE & 068 & 61.8 \\
\hline & MULTIPLE & 028 & 25.5 \\
\hline & NOT SPECIFIED & 014 & 12.7 \\
\hline & Total & 108 & 100.0 \\
\hline \multirow{3}{*}{ Healing } & NO HEALING & 026 & 23.6 \\
\hline & HEALING & 084 & 76.4 \\
\hline & Total & 109 & 100.0 \\
\hline
\end{tabular}


With regards to the characteristics of the ORN found, $9(8.2 \%)$ of the articles did not specify the location of the lesion. Out of the 81 articles with a single lesion, 2 of them presented in the maxilla $(1.8 \%), 79$ in the jaw (71.8\%), and $20(18.2 \%)$ presented in both jaws. With regards to the number of lesions, $61.8 \%$ of the articles described single lesions, $25.5 \%$ described multiple simultaneous lesions, and in $12.7 \%$ of the articles this was not specified. In $22.7 \%$ of the cases, the lesions appeared solely in the posterior region, however in $33.6 \%$ of the cases these appeared both in the anterior and posterior sectors.

As far as the mean onset time, there was a significant variability in with a range from 2 months to 183 months, however the mean was 45.7 months $(\mathrm{SD}=36.2)$, that is to say 3.8 years. Evidently the appearance of the lesions depends on the maximum follow-up time, which, in this systematic review was broad and variable, ranging from 6 to 444 months, with a mean of 58.9 months $(\mathrm{SD}=76.5)$. In terms of the therapeutic alternatives used, the surgical treatment was the most common choice representing $45.5 \%$ of cases, and pharmacological treatment, exclusively or combined, was the least common, with $26.1 \%$ and $28.4 \%$ of cases respectively. Only one study, that is to say $0.90 \%$ corresponded to the treatment of ORN by LLLT. Radical surgical treatment with free flap was the most used surgical alternative in $60.2 \%$ of the cases, followed by debridement (curettage and/or sequestrectomy or marginal resection) in $22.7 \%$. In terms of exclusively pharmacological treatments, HBO accounted for $58.3 \%$, followed by the use of pentoxifylline, with or without clodronate in $21.6 \%$ of the cases. The systematic review shows an overall healing of $77.2 \%$ of the lesions.

The healing of the ORN lesions is understood as the absence of relapse during the follow-up period, which as shown before, is very variable. This healing appears to vary depending on the type of treatment performed. Out of 88 cases which were treated by surgical intervention, only $73.7 \%$ of the cases were cured, and likewise, $70.0 \%$ of the 60 cases, which were treated by pharmacological means were cured. Broadly speaking, treatment exclusively by surgical intervention seems to be effective option, with $51.2 \%$ of the lesions healed, whereas only $28.6 \%$ of the lesions of patients who were treated exclusively by pharmacological means, and $17.9 \%$ of the lesions in patients who underwent combined medicalsurgical treatment $(p=0.002)$ were healed. In the study conducted with LLLT therapy combined with antimicrobial photodynamic therapy (aPDT), 20 patients were treated and $100 \%$ of the patients were cured.

Statistically significant differences between healing and the type of surgical treatment were not observed, however, as we can see in Table 4, statistically significant differences were observed when using pharmacological treatment, Table 4. Pentoxifylline with/without clodronate made a major and significant contribution to the healing in $84,6 \%$ of the cases where was used, with HBO healed in $62.8 \%$ of the cases, whereas other alternatives, such as the exclusive use of antibiotics/ anti-inflammatories/antiseptics failed in $100 \%$ of the patients $(p=0.043)$. By performing a binomial logistic regression analysis, we verified that the type of treatment is the only statistically significant factor related to healing. Therefore, taking the combined medical-surgical treatment as a reference, exclusive surgical treatment shows an OR for healing of 5.7 (CI95\% 1.9-16.9, $p=0.002$ ) and 5.7 for pharmacological treatment (CI95\% $1.5-20.2, p=0.009$ ). Given that only one study was treated with LLLT, this has been excluded from the equation.

Table 4: Comparison of the healing process according to the different types of treatment gathered for ORN. AAA (Antibiotics, anti-inflammatories, antiseptics); PRGF (platelet rich growth factor).

\begin{tabular}{|c|c|c|c|c|c|c|c|c|}
\hline \multirow{2}{*}{ Free flap } & \multicolumn{6}{|c|}{ Surgical treatment } & \multirow{2}{*}{ Total } & \multirow{2}{*}{$p$ value } \\
\hline & $\begin{array}{c}\text { Pediculated } \\
\text { soft tissue }\end{array}$ & $\begin{array}{c}\text { Artificial } \\
\text { dermal skin }\end{array}$ & $\begin{array}{c}\text { Debride- } \\
\text { ment }\end{array}$ & Block graft & \begin{tabular}{|l|} 
Segmental \\
Osteotomy
\end{tabular} & & & \\
\hline NO HEALING & $12(52.2 \%)$ & $2(8.7 \%)$ & 0 & $6(26.1 \%)$ & 0 & $3(13.0 \%)$ & $23(100.0 \%)$ & 0.811 \\
\hline HEALING & $41(63.1 \%)$ & $4(6.2 \%)$ & $1(1.5 \%)$ & $14(21.5 \%)$ & $1(1.5 \%)$ & $4(6.2 \%)$ & $65(100.0 \%)$ & \\
\hline Total & $53(60.2 \%)$ & $6(6.8 \%)$ & $1(1.1 \%)$ & $20(22.7 \%)$ & $1(1.1 \%)$ & $7(7.9 \%)$ & $88(100.0 \%)$ & \\
\hline \multirow{2}{*}{ Pentoxifylline } & \multicolumn{6}{|c|}{ Pharmacological treatment } & \multirow{2}{*}{ Total } & \multirow{2}{*}{$p$ value } \\
\hline & AAA & PRGF & HBO & Teriparatide & Combined & & & \\
\hline NO HEALING & $2(11.1 \%)$ & $2(11.1 \%)$ & 0 & $13(72.2 \%)$ & 0 & $1(5.5 \%)$ & $18(100.0 \%)$ & 0.043 \\
\hline HEALING & $11(26.2 \%)$ & 0 & $5(11.9 \%)$ & $22(52.4 \%)$ & $2(4.8 \%)$ & $2(4.8 \%)$ & $42(100.0 \%)$ & \\
\hline Total & $13(21.6 \%)$ & $2(3.3 \%)$ & $5(8.3 \%)$ & $35(58.3 \%)$ & $2(3.3 \%)$ & $3(5.0 \%)$ & $60(100.0 \%)$ & \\
\hline
\end{tabular}




\section{Discussion}

ORN is a serious complication which is difficult and expensive to treat (21). In order to manage the disease in its early stages, the treatment must be conservative. The authors recommend oral hygiene, optimisation of the nutritional condition and a multidisciplinary management, which includes minor surgery, how the dental extraction, or debridement of the necrotic tissue and antibiotics (28).

In the 1960s, after its implementation by Marx (9), HBO began to be used as an additional treatment for ORN, as a complement for soft tissue flaps and in the management of radiated tissues. Although HBO initially showed promising results in the treatment of ORN (11), today's literature shows very disparate results in the use of this technique (29).

In the advanced stages or recurrences of the disease, a surgical reconstruction of the jaw is performed by means of the surgical resection and immediate transfer of the tissue to the disease, especially in stage III (30). The reconstruction of a free flap in the radiated jaw is difficult. The identification and dissection of the receiving vessels can be arduous and it requires for vessels to be selected from outside of the radiated field, generally from the contralateral neck (13).

Furthermore, it is an expensive procedure, due to hospital stay (21). Recently factors like appearance, swallowing, and chewing that interfere with the quality of life were analyzed and showed that the approach with adequate debridement, resection, and reconstruction may greatly improve QOL (31). The surgical treatments identified in the studies include sequestrectomy and debridement (32), free flap (33), pediculate soft tissue (34) and block grafting (35).

In this review, $21.6 \%$ of the studies presented ORN cases, which were treated with pentoxifylline and PENTOCLO. This management is used in both the early and advanced stages of the disease. The combined medical therapy showed a recovery rate of $88.9 \%$ in the 13 presented studies, and in just $11.1 \%$ of them ( 2 studies), the disease progressed and subsequent surgery was necessary for healing. Some of these studies presented patients whose recovery had already failed with other conservative therapies, such as the study conducted by Delanian (22), in which 16 out of 18 patients completely recovered and, out of these, 14 were fully recovered within 7 months. In 2011 (36), a subsequent study conducted by the same researchers on refractory ORN of the jaw treated by means of HBO and surgical intervention, studied the combination of pentoxifylline and vitamin E, together with clodronate, antibiotics and steroids as treatment. All of the patients (100\%) presented with a complete regression of the exposed bone and were fully recovered within 2 years after treatment, with 50 $\%$ of the patients recovering in just 6 months.
In research performed by D'Souza (7), the results of ORN patients who had received medical treatment with pentoxifylline, tocopherol and doxycycline were compared with those of patients who had been treated with HBO. $25 \%$ and $51 \%$ of the patients respectively showed a progression of the disease and required free flap reconstruction. Furthermore, in the group of patients that received medical treatment there were no recurrences of ORN following the resection and the free flap reconstruction, in comparison with a $20 \%$ recurrence in the group treated with HBO. This confirms the current understanding of the pathophysiology of ORN based on the fibrosis induced by radiation.

Recently, other alternatives for the management of ORN have been discussed in the literature, and these include plasmatic factors modified in all their versions (PRGF, PLT-gel, L-PRF), Teriparatide and LLLT. With regards to plasma rich in growth factors (PRGF), its use was suggested following reports in which it was demonstrated that its application as filling material in surgeries and pre-prosthetic implants presented excellent adjuvant and regenerative proprieties (37). The RIF process reduces the level of expression of the transforming growth factor beta (TGF- $\beta$ ). The use of PRGF formulations is based on the premise that the growth factors contained in platelet granules, which are released after activation are beneficial to improving the tissue regeneration (37). In a study, which was performed by Gallesio on 10 patients (38), on day 14 after surgery, the treated area presented complete wound closure.

Cha (23), presented a study in which Teriparatide -a recombinant human parathyroid hormone- was used, demonstrating its beneficial effects on bone regeneration of ORN of the jaw in advanced stages. However, the studies performed on rat models have shown a theoretical risk of osteosarcoma, therefore confirming the need for further studies (39).

The only LLLT report found in our review dated back to 2018 (24). The effectiveness of LLLT is supported by studies in which its effects on the healing process of the oral mucosa are highlighted. These studies have also demonstrated that it minimises the exudative phase, boosts healing and leads to the proliferation and transformation of fibroblasts and myofibroblasts that help in tissue repair, due to the release of growth factors (40). Ribeiro (24) presented a protocol for management with LLLT, in which the 20 treated patients presented with the pathology in early to advanced stages. $100 \%$ of the reported cases were healed with no recurrence during the two follow-up years. This therapy is also non-invasive, atraumatic and no significant associated adverse effects have been reported in the literature.

Among the limitations to this systematic review, it is important to mention that it mostly consists of a retrospective group of cases and case reports, therefore mean- 
ing that their heterogeneous nature and the absence of randomised trials is a limiting factor. As a consequence of these disadvantages, the possibility of carrying out a more objective analysis in which more powerful conclusions are drawn would prove challenging.

The results obtained out of all of the different treatments proposed for ORN, seem to indicate that the combined surgical and / or pharmacological treatment (PENTOCLO), is the treatment of choice and offers better healing rates. In case of recurrence, there is some evidence that resection surgery and reconstruction may also be considered, respecting the particular circumstances in which each should be used. What seems clear is that early intervention with conservative surgical and pharmacological methods improves the prognosis of ORN. In an attempt to expand less invasive treatment methods, we suggest more studies for conservative surgical management of hard tissue associated with LLLT therapy, based on controlled clinical studies, with welldistinguished control groups are necessary in order to establish a more efficient therapeutic pattern.

\section{References}

1. Ray-Chaudhuri A, Shah K, Porter RJ. The oral management of patients who have received radiotherapy to the head and neck region. Br Dent J. 2013;214:387-93.

2. Abed H, Reilly D, Burke M, Daly B. Patients with head and neck cancers' oral health knowledge, oral health-related quality of life, oral health status, and adherence to advice on discharge to primary dental care: A prospective observational study. Observational Study. 2019;39:593-602.

3. Kim BJ, Kang HG, Lee SW, Jung J, Lee MH, Kang DW, et al. Changes in the Common Carotid Artery after Radiotherapy: Wall Thickness, Calcification, and Atherosclerosis. J Clin Neurol. 2018;14:35-42.

4. Murthy V, Gupta T, Kadam A, Ghosh-Laskar S, Budrukkar A, Phurailatpam R, et al. Time trial: A prospective comparative study of the time-resource burden for three-dimensional conformal radiotherapy and intensity-modulated radiotherapy in head and neck cancers. J Cancer Res Ther. 2009;5:107-12.

5. Clark CH, Miles EA, Urbano MTG, Bhide SA, Bidmead AM, Harrington $\mathrm{KJ}$, et al. Pre-trial quality assurance processes for an intensity-modulated radiation therapy (IMRT) trial: PARSPORT, a UK multicentre Phase III trial comparing conventional radiotherapy and parotid-sparing IMRT for locally advanced head and neck cancer. $\mathrm{Br}$ J Radiol. 2009 Jul;82:585-94.

6. Nabil S, Ramli R. The use of buccal fat pad flap in the treatment of osteoradionecrosis. Int J Oral Maxillofac Surg. 2012;41:1422-6.

7. D'Souza J, Lowe D, Rogers SN. Changing trends and the role of medical management on the outcome of patients treated for osteoradionecrosis of the mandible: Experience from a regional head and neck unit. Br J Oral Maxillofac Surg. 2014;52:356-62.

8. Lyons AJ, West CM, Risk JM, Slevin NJ, Chan C, Crichton S, et al. Osteoradionecrosis in Head-and-Neck Cancer Has a Distinct Genotype-Dependent Cause. Int J Radiat Oncol. 2012;82:1479-84.

9. Marx RE. Osteoradionecrosis: A new concept of its pathophysiology. J Oral Maxillofac Surg. 1983;41:283-8.

10. Epstein JB, Wong FL, Stevenson-Moore P. Osteoradionecrosis: clinical experience and a proposal for classification. J Oral Maxillofac Surg. 1987;45:104-10.

11. Mainous EG. Hyperbaric oxygen in total rehabilitation of patients with mandibular osteoradionecrosis. Int J Oral Surg. 1974;3:297-301.

12. Santamaria E, Wei FC, Chen HC. Fibula osteoseptocutaneous flap for reconstruction of osteoradionecrosis of the mandible. Plast Reconstr Surg. 1998;101:921-9.

13. Ang E, Black C, Irish J, Brown DH, Gullane P, O’Sullivan B, et al. Reconstructive options in the treatment of osteoradionecrosis of the craniomaxillofacial skeleton. Br J Plast Surg. 2003;56:92-9.

14. Marx RE, Johnson RP. Studies in the radiobiology of osteoradionecrosis and their clinical significance. Oral Surg Oral Med Oral Pathol. 1987;64:379-90.

15. London SD, Park SS, Gampper TJ, Hoard MA. Hyperbaric oxygen for the management of radionecrosis of bone and cartilage. Laryngoscope. 1998;108:1291-6.

16. Lyons A, Osher J, Warner E, Kumar R, Brennan PA. Osteoradionecrosis - A review of current concepts in defining the extent of the disease and a new classification proposal. Br J Oral Maxillofac Surg. 2014;52:392-5.

17. Epstein JB, Robertson M, Emerton S, Phillips N, StevensonMoore P. Quality of life and oral function in patients treated with radiation therapy for head and neck cancer. Head Neck. 2001;23:389-98. 18. Shaw RJ, Butterworth C. Hyperbaric oxygen in the management of late radiation injury to the head and neck. Part II: prevention. Br J Oral Maxillofac Surg. 2011;49:9-13.

19. McLeod NMH, Bater MC, Brennan PA. Management of patients at risk of osteoradionecrosis: results of survey of dentists and oral \& maxillofacial surgery units in the United Kingdom, and suggestions for best practice. Br J Oral Maxillofac Surg. 2010;48:301-4.

20. Delanian S, Lefaix JL. The radiation-induced fibroatrophic process: therapeutic perspective via the antioxidant pathway. Radiother Oncol. 2004;73:119-31.

21. Patel V, Ormondroyd L, Lyons A, McGurk M. The financial burden for the surgical management of osteoradionecrosis. Br Dent J. 2017;222:177-80.

22. Delanian S, Depondt J, Lefaix JL. Major healing of refractory mandible osteoradionecrosis after treatment combining pentoxifylline and tocopherol: A phase II trial. Head Neck. 2005;27:114-23.

23. Cha YH, Hong N, Rhee Y, Cha IH. Teriparatide therapy for severe, refractory osteoradionecrosis of the jaw. Osteoporos Int. 2018;29:987-92.

24. Ribeiro GH, Minamisako MC, Rath IBD, Santos AMB, Simoes A, Pereira KCR, et al. Osteoradionecrosis of the jaws: case series treated with adjuvant low-level laser therapy and antimicrobial photodynamic therapy. J Appl Oral Sci. 2018;26:e20170172.

25. Liberati A, Altman DG, Tetzlaff J, Mulrow C, Gøtzsche PC, Ioannidis JPA, et al. The PRISMA statement for reporting systematic reviews and meta-analyses of studies that evaluate health care interventions: explanation and elaboration. PLoS Med. 2009;6:e1000100. 26. Stang A. Critical evaluation of the Newcastle-Ottawa scale for the assessment of the quality of nonrandomized studies in metaanalyses. Eur J Epidemiol. 2010;25:603-5.

27. Frykberg RG, Banks J. Challenges in the Treatment of Chronic Wounds. Adv wound care. 2015;4:560-82.

28. Chronopoulos A, Zarra T, Tröltzsch M, Mahaini S, Ehrenfeld M, Otto S. Osteoradionecrosis of the mandible: A ten year single-center retrospective study. J Cranio-Maxillofacial Surg. 2015;43:837-46.

29. Sultan A, Hanna GJ, Margalit DN, Chau N, Goguen LA, Marty FM, et al. The Use of Hyperbaric Oxygen for the Prevention and Management of Osteoradionecrosis of the Jaw: A Dana-Farber/ Brigham and Women's Cancer Center Multidisciplinary Guideline. Oncologist. 2017;22:343-50.

30. Jacobson AS, Buchbinder D, Urken ML. Reconstruction of bilateral osteoradionecrosis of the mandible using a single fibular free flap. Laryngoscope. 2010;120:273-5.

31. Danielsson D, Munck-Wikland E, Hagel E, Halle M. Quality of life after microvascular mandibular reconstruction for osteoradionecrosis-A prospective study. Head Neck. 2019;41:2225-30.

32. Mucke T, Koschinski J, Rau A, Loeffelbein DJ, Deppe H, Mitchell DA, et al. Surgical outcome and prognostic factors after treatment of osteoradionecrosis of the jaws. J Cancer Res Clin Oncol. 2013;139:389-94.

33. Chang DW, Oh HK, Robb GL, Miller MJ. Management of ad- 
vanced mandibular osteoradionecrosis with free flap reconstruction. Head Neck. 2001;23:830-5.

34. Woo SH. Buccinator Myomucosal Flap for Treatment of Osteoradionecrosis of the Mandible. Clin Exp Otorhinolaryngol. 2016;9:85-8. 35. Etezadi A, Ferguson H, Emam HA, Walker P. Multiple Remediation of Soft Tissue Reconstruction in Osteoradionecrosis of the Mandible: A Case Report. J Oral Maxillofac Surg. 2013;71:e1-6.

36. Delanian S, Chatel C, Porcher R, Depondt J, Lefaix JL. Complete restoration of refractory mandibular osteoradionecrosis by prolonged treatment with a pentoxifylline-tocopherol-clodronate combination (PENTOCLO): A phase II trial. Int J Radiat Oncol Biol Phys. 2011;80:832-9.

37. Batstone MD, Cosson J, Marquart L, Acton C. Platelet rich plasma for the prevention of osteoradionecrosis. A double blinded randomized cross over controlled trial. Int J Oral Maxillofac Surg. 2012;41:2-4

38. Gallesio G, Del Fabbro M, Pol R, Mortellaro C, Mozzati M. Conservative treatment with plasma rich in growth factors-Endoret for osteoradionecrosis. J Craniofac Surg. 2015;26:731-6.

39. Andrews EB, Gilsenan A, Midkiff K, Harris D. Challenges in studying very rare cancer outcomes and infrequent exposures: example of teriparatide and osteosarcoma. Ann Epidemiol. 2016;26:751-3. 40. Woodruff LD, Bounkeo JM, Brannon WM, Dawes KS, Barham $\mathrm{CD}$, Waddell DL, et al. The efficacy of laser therapy in wound repair: a meta-analysis of the literature. Photomed Laser Surg. 2004;22:241-7.

\section{Funding}

None declared.

\section{Conflict of interest}

None declared.

\section{Authors contributions}

Gisela CV Camolesi: Was responsible for acquisition of data: literature search, analysis and interpretation of data collected and drafting of article.

Karem L. Ortega: Was responsible for the final approval of manuscript.

Janaina Braga Medina: Was responsible for the final approval of manuscript.

Luana Campos: Was responsible for the analysis and interpretation of data collected, and final approval of manuscript.

Alejandro I Lorenzo Pouso: Was responsible for the final approval of manuscript.

Pilar Gándara Vila: Was responsible for the final approval of manuscript.

Mario Pérez Sayáns: Was responsible for conception and design of review, acquisition of data: literature search, analysis and interpretation of data collected, drafting of article and/or critical revision, final approval of manuscript 